\section{¿Menos teléfonos, más libertades?}

Less phones, more freedoms?

\section{Sr. Editor}

El viernes diez de mayo comenzó la restricción del uso de teléfonos móviles al interior de un recinto escolar en Calama. Esto, luego de que un caso de ciberbullying llevara al Tribunal de Familia local a dictaminar el mandato. Al respecto surge naturalmente la pregunta: ¿Es justificada una medida restrictiva de este tipo?

Resulta que las redes sociales tienen efectivamente un rol en la exposición al ciberbullying, mientras que este a su vez aumenta el riesgo de autolesiones, ideación e intentos suicidas, no sólo en la víctima, sino también en el victimario ${ }^{1}$. Cabe recordar que reducir el suicidio adolescente es parte de los objetivos sanitarios de la década determinados por el Ministerio de Salud.

Mientras un mayor tiempo en redes sociales se ha correlacionado con un peor bienestar psicológico autorreportado, así como con ideaciones suicidas, Chile lidera la OCDE como el país en que más ha aumentado el uso de internet en escolares fuera del horario de clases, situación que -es razonable asumir- se repite en las aulas ${ }^{2}$. Por su parte, investigaciones revelan que restringir el uso de teléfonos podría disminuir significativamente la percepción de soledad, así como de depresión, ansiedad y sensación de "missing out"3.

El efecto potencial de una intervención como esta podría no limitarse únicamente al bienestar psicológico de los menores: La distracción con notificaciones disminuye el placer que la actividad original en desarrollo genera, y eliminar la disponibilidad de teléfonos ha demostrado incrementar la densidad de toma de apuntes, así como el nivel de retención de contenido y, eventualmente, del desempeño de los estudiantes ${ }^{4,5}$.

Entonces, ¿es justificada la medida? La verdad es que, considerando que algunos plantean el uso intencionado de estos aparatos en las aulas, no tenemos tanta certeza como quisiéramos al respecto. Lo que si sabemos es que investigaciones recientes apoyan la tesis que guiaría tal toma de decisión, y que restricciones similares han sido implementadas exitosamente en otras partes del globo. Quizás lo que debemos en este caso es aprovechar la oportunidad y tomar el acontecer del liceo de Calama como caso de estudio local, generando evidencia que permita luego replicar o retractar tal decisión.

\section{Referencias}

1. John A, Glendenning AC, Marchant A, Montgomery P, Stewart A, Wood S, et al. Self-Harm, Suicidal Behaviours, and Cyberbullying in Children and Young People: Systematic Review. Journal of Medical Internet Research. 19 de abril de 2018;20(4):9

2. Memon AM, Sharma SG, Mohite SS, Jain S. The role of online social networking on deliberate selfharm and suicidality in adolescents: A systematized review of literature. Social networking. 2018;60(4):9.

3. Hunt MG, Marx R, Lipson C, Young J. No More FOMO: Limiting Social Media Decreases Loneliness and Depression. Journal of Social and Clinical Psychology. diciembre de 2018;37(10):751-68.

4. Isikman E, MacInnis DJ, Ülkümen G, Cavanaugh LA. The effects of curiosity-evoking events on activity enjoyment. Journal of Experimental Psychology: Applied. septiembre de 2016;22(3):319-30.

5. Kuznekoff JH, Titsworth S. The Impact of Mobile Phone Usage on Student Learning. Communication Education. julio de 2013;62(3):233-52.

Claudio Daza de la Parra Pontificia Universidad Católica de Chile

Correspondencia: 